\title{
ORIGINAL
}

\author{
Yuriy Povstenko
}

\section{Time-fractional radial heat conduction in a cylinder and associated thermal stresses}

Received: 7 August 2010 / Accepted: 8 June 2011 / Published online: 23 June 2011

(C) The Author(s) 2011. This article is published with open access at Springerlink.com

\begin{abstract}
The theory of thermal stresses based on the heat conduction equation with the Caputo time-fractional derivative of order $0<\alpha \leq 2$ is used to investigate axisymmetic thermal stresses in a cylinder. The solution is obtained applying the Laplace and finite Hankel integral transforms. The Dirichlet and two types of Neumann problems with the prescribed boundary value of the temperature, the normal derivative of the temperature, and the heat flux are considered. Numerical results are illustrated graphically.
\end{abstract}

Keywords Fractional calculus · Mittag-Leffler functions $\cdot$ Non-Fourier heat conduction $\cdot$ Thermal stresses

\section{Introduction}

The classical theory of thermoelasticity investigates stresses caused by the temperature field found from the standard parabolic heat conduction equation. In non-classical theories, this equation is replaced by more general one leading to generalized theories of thermoelasticity. For an extensive bibliography on this subject and further discussion see for example, [1-7] and references therein.

For a cylinder, a lot of problems describing interesting phenomena that characterize different theories of thermoelasticity have been solved by many researchers (see e.g. [8-19]).

The time-nonlocal dependence between the heat flux vector and the temperature gradient with the "longtale" power kernel can be interpreted in terms of fractional integrals and derivatives [20-23]:

$$
\begin{array}{ll}
\mathbf{q}(t)=-k D_{R L}^{1-\alpha} \operatorname{grad} T(t), & 0<\alpha \leq 1, \\
\mathbf{q}(t)=-k I^{\alpha-1} \operatorname{grad} T(t), & 1<\alpha \leq 2,
\end{array}
$$

where $D_{R L}^{1-\alpha}$ and $I^{\alpha-1}$ are the Riemann-Liouville fractional derivative and integral, respectively. The constitutive Eq. (1) yields the time-fractional heat conduction equation with the Caputo derivative of order $0<\alpha \leq 2$. A quasi-static uncoupled theory of thermoelasticity based on fractional heat conduction equation was put forward by Povstenko [20]. In the framework of this theory, two-dimensional axisymmetric stress in an infinite space was considered in [24]. A medium with a cylindrical hole was investigated in [25]. In the present article, in the framework of fractional thermoelasticity, we study axisymmetric thermal stresses in a cylinder. The interested reader is also referred to the previous paper [26], where several problems for fractional heat conduction equation in a cylinder were solved under various conditions for the temperature. Time-fractional heat conduction (or diffusion) in a cylinder was also studied in [27-32]. Here, we consider the Dirichlet problem with the prescribed boundary value of the temperature and two types of the Neumann problem: the first

Y. Povstenko $(\varangle)$

Institute of Mathematics and Computer Science, Jan Długosz University in Częstochowa,

al. Armii Krajowej 13/15, 42-200 Czestochowa, Poland

E-mail: j.povstenko@ajd.czest.pl 
Neumann problem with the given boundary value of the normal derivative of temperature and the second one with the prescribed boundary value of the heat flux. In the case of classical heat conduction equation, these two types of Neumann problem are identical. Numerical results show unusual behavior of solutions to fractional heat conduction equation as well as the associated stresses in comparison with the corresponding solutions obtained in the framework of the classical theory.

It should be also emphasized that in the framework of quasi-static theory taking coupling between deformation and heat conduction in the heat equation into account does not complicate the Neumann bounadry value problem, but only slightly changes constant coefficient.

\section{Formulation of the problem}

A theory of thermal stresses is governed by the equation of motion in terms of displacements

$$
\mu \Delta \mathbf{u}+(\lambda+\mu) \operatorname{grad} \operatorname{div} \mathbf{u}-\rho \frac{\partial^{2} \mathbf{u}}{\partial t^{2}}=\beta K \operatorname{grad} T,
$$

the stress-strain-temperature relation

$$
\sigma=2 \mu \mathbf{e}+(\lambda \operatorname{tr} \mathbf{e}-\beta K T) \mathbf{I},
$$

and the time-fractional heat conduction equation

$$
\frac{\partial^{\alpha} T}{\partial t^{\alpha}}+\gamma_{e} \frac{\partial^{\alpha} \operatorname{tr} \mathbf{e}}{\partial t^{\alpha}}=a \Delta T, \quad 0<\alpha \leq 2
$$

where body forces are neglected, $\mathbf{u}$ is the displacement vector, $\sigma$ the stress tensor, $\mathbf{e}$ the linear strain tensor, $T$ the temperature, $\lambda$ and $\mu$ are Lamé constants, $K=\lambda+2 \mu / 3, \beta$ is the thermal coefficient of volumetric expansion, and $\mathbf{I}$ denotes the unit tensor. For $\alpha=1$, the coefficient $a$ denotes the thermal diffusivity. The coefficient $\gamma_{e}$ describes the effect of deformation on the thermal state of a solid.

In Eq. (4), $\frac{\partial^{\alpha} T}{\partial t^{\alpha}}$ is the Caputo fractional derivative (see e.g. [33,34]):

$$
\frac{\mathrm{d}^{\alpha} f(t)}{\mathrm{d} t^{\alpha}}= \begin{cases}\frac{1}{\Gamma(n-\alpha)} \int_{0}^{t}(t-\tau)^{n-\alpha-1} \frac{\mathrm{d}^{n} f(\tau)}{\mathrm{d} \tau^{n}} \mathrm{~d} \tau, & n-1<\alpha<n, \\ \frac{\mathrm{d}^{n} f(t)}{\mathrm{d} t^{n}}, & \alpha=n,\end{cases}
$$

with the following Laplace transform rule

$$
\mathcal{L}\left\{\frac{\mathrm{d}^{\alpha} f(t)}{\mathrm{d} t^{\alpha}}\right\}=s^{\alpha} \mathcal{L}\{f(t)\}-\sum_{k=0}^{n-1} f^{(k)}\left(0^{+}\right) s^{\alpha-1-k}, \quad n-1<\alpha<n,
$$

where $s$ is the transform variable.

In what follows we consider the quasi-static theory neglecting the inertia term in Eq. (2). From physical point of view, this means that no account has been taken of mechanical oscillations.

In the case of axial symmetry, the equation of equilibrium (2) is rewritten as

$$
\frac{\partial \operatorname{tr} \mathbf{e}}{\partial r}=m \frac{\partial T}{\partial r}
$$

or

$$
\frac{\partial^{2} u}{\partial r^{2}}+\frac{1}{r} \frac{\partial u}{\partial r}-\frac{u}{r^{2}}=m \frac{\partial T}{\partial r}
$$

where $m=\frac{1+v}{1-v} \frac{\beta}{3}$ with $v$ being the Poisson ratio.

It is well known (see e.g. [35]) that in the framework of quasi-static approach, the coupling term $\gamma_{e} \frac{\partial^{\alpha} \operatorname{tr} \mathbf{e}}{\partial t^{\alpha}}$ can be eliminated from Eq. (4) in an unbounded solid resulting in modification of the coefficient: $a^{\prime}=a /\left(1+m \gamma_{e}\right)$. Similarly, in a bounded solid, the coupling term in Eq. (4) can also be eliminated, but the boundary conditions 
become couple. Equation (7) shows that for the considered geometry in the case of Neumann problem, the coupling term can also be eliminated from the boundary condition, only slightly changing the constant coefficient by a factor $1 /\left(1+m \gamma_{e}\right)$. Hence, in what follows we consider uncoupled theory.

The surface of a cylinder is traction free so that

$$
r=R: \quad \sigma_{r r}=0 .
$$

Equation (8) has the solution (see e.g. Parkus [36]):

$$
u=C_{1} r+\frac{C_{2}}{r}+\frac{m}{r} \int_{0}^{r} x T(x, t) \mathrm{d} x .
$$

The integration constants are found from the boundary and boundedness conditions:

$$
C_{1}=(1-2 v) m \frac{1}{R^{2}} \int_{0}^{R} x T(x, t) \mathrm{d} x, \quad C_{2}=0 .
$$

It follows from Eqs. (3), (10), and (11) that the stress tensor components can be calculated as integrals of the temperature field $[36,37]$

$$
\begin{aligned}
& \sigma_{r r}(r, t)=2 \mu m\left[\frac{1}{R^{2}} \int_{0}^{R} x T(x, t) \mathrm{d} x-\frac{1}{r^{2}} \int_{0}^{r} x T(x, t) \mathrm{d} x\right], \\
& \sigma_{\theta \theta}(r, t)=2 \mu m\left[\frac{1}{R^{2}} \int_{0}^{R} x T(x, t) \mathrm{d} x+\frac{1}{r^{2}} \int_{0}^{r} x T(x, t) \mathrm{d} x-T(r, t)\right] .
\end{aligned}
$$

For plane strain with constant axial strain, we obtain

$$
\sigma_{z z}(r, t)=\sigma_{r r}(r, t)+\sigma_{\theta \theta}(r, t)=2 \mu m\left[\frac{2}{R^{2}} \int_{0}^{R} x T(x, t) \mathrm{d} x-T(r, t)\right] .
$$

\section{Solution to the Dirichlet problem}

For a cylinder in the case of axial symmetry, the heat conduction Eq. (4) is rewritten as

$$
\frac{\partial^{\alpha} T}{\partial t^{\alpha}}=a\left(\frac{\partial^{2} T}{\partial r^{2}}+\frac{1}{r} \frac{\partial T}{\partial r}\right), \quad 0 \leq r<R, \quad 0<t<\infty .
$$

The zero initial conditions are assumed

$$
\begin{array}{ll}
t=0: & T=0, \quad 0<\alpha \leq 2, \\
t=0: & \frac{\partial T}{\partial t}=0, \quad 1<\alpha \leq 2 .
\end{array}
$$

Equation (15) should be also subjected to the appropriate boundary condition. Consider the Dirichlet problem with the prescribed boundary value of temperature:

$$
r=R: T=p(t) .
$$

Applying the Laplace transform with respect to time $t$ and the finite Hankel transform with respect to the polar coordinate $r$ (see "Appendix"), we obtain

$$
T^{* *}\left(\xi_{i}, s\right)=a R \xi_{i} J_{1}\left(R \xi_{i}\right) \frac{1}{s^{\alpha}+a \xi_{i}^{2}} p^{*}(s),
$$

where each integral transform is denoted by an asterisk. 
Inversion of the integral transforms with using the convolution theorem and taking into account that $[34,38]$

$$
\mathcal{L}^{-1}\left\{\frac{s^{\alpha-\beta}}{s^{\alpha}+a \xi_{i}^{2}}\right\}=t^{\beta-1} E_{\alpha, \beta}\left(-a \xi_{i}^{2} t^{\alpha}\right)
$$

leads to

$$
T(r, t)=\frac{2 a}{R} \sum_{i=1}^{\infty} C_{\alpha, \alpha}\left(\xi_{i}, t\right) \frac{\xi_{i} J_{0}\left(r \xi_{i}\right)}{J_{1}\left(R \xi_{i}\right)}
$$

where

$$
C_{\alpha, \alpha}\left(\xi_{i}, t\right)=\int_{0}^{t} \tau^{\alpha-1} E_{\alpha, \alpha}\left(-a \xi_{i}^{2} \tau^{\alpha}\right) p(t-\tau) \mathrm{d} \tau,
$$

$E_{\alpha, \beta}(z)$ are the generalized Mittag-Leffler functions in two parameters [34,39]:

$$
E_{\alpha, \beta}(z)=\sum_{n=0}^{\infty} \frac{z^{n}}{\Gamma(\alpha n+\beta)}, \quad \alpha>0, \beta>0, z \in C .
$$

Integration of temperature according to (12)-(14) gives

$$
\begin{aligned}
\sigma_{r r}(r, t) & =\frac{4 \mu m a}{r R^{2}} \sum_{i=1}^{\infty} C_{\alpha, \alpha}\left(\xi_{i}, t\right) \frac{r J_{1}\left(R \xi_{i}\right)-R J_{1}\left(r \xi_{i}\right)}{J_{1}\left(R \xi_{i}\right)} \\
\sigma_{\theta \theta}(r, t) & =\frac{4 \mu m a}{r R^{2} r} \sum_{i=1}^{\infty} C_{\alpha, \alpha}\left(\xi_{i}, t\right) \frac{r J_{1}\left(R \xi_{i}\right)+R J_{1}\left(r \xi_{i}\right)-r R \xi_{i} J_{0}\left(r \xi_{i}\right)}{J_{1}\left(R \xi_{i}\right)} \\
\sigma_{z z}(r, t) & =\frac{4 \mu m a}{R^{2}} \sum_{i=1}^{\infty} C_{\alpha, \alpha}\left(\xi_{i}, t\right) \frac{2 J_{1}\left(R \xi_{i}\right)-R \xi_{i} J_{0}\left(r \xi_{i}\right)}{J_{1}\left(R \xi_{i}\right)}
\end{aligned}
$$

Now let us consider the particular case of the obtained solution corresponding to the constant boundary value of temperature:

$$
r=R: \quad T=T_{0}=\text { const. }
$$

In this instance

$$
T^{* *}\left(\xi_{i}, s\right)=a R T_{0} \xi_{i} J_{1}\left(R \xi_{i}\right) \frac{1}{s\left(s^{\alpha}+a \xi_{i}^{2}\right)} .
$$

Since

$$
\frac{1}{s\left(s^{\alpha}+a \xi_{i}^{2}\right)}=\frac{1}{a \xi_{i}^{2}}\left(\frac{1}{s}-\frac{s^{\alpha-1}}{s^{\alpha}+a \xi_{i}^{2}}\right)
$$

and

$$
\mathcal{L}^{-1}\left\{\frac{s^{\alpha-1}}{s^{\alpha}+a \xi_{i}^{2}}\right\}=E_{\alpha}\left(-a \xi_{i}^{2} t^{\alpha}\right),
$$

the inverse of Eq. (28) with taking into account (A.7) assumes the form

$$
T=T_{0}-2 T_{0} \sum_{i=1}^{\infty} E_{\alpha}\left(-a \xi_{i}^{2} t^{\alpha}\right) \frac{J_{0}\left(r \xi_{i}\right)}{R \xi_{i} J_{1}\left(R \xi_{i}\right)},
$$


where

$$
E_{\alpha}(z)=\sum_{n=0}^{\infty} \frac{z^{n}}{\Gamma(\alpha n+1)}, \quad \alpha>0, z \in C,
$$

is the Mittag-Leffler function in one parameter.

In terms of dimensionless quantities

$$
\bar{T}=\frac{T}{T_{0}}, \quad \bar{\sigma}_{i j}=\frac{\sigma_{i j}}{2 \mu m T_{0}}, \quad \rho=\frac{r}{R}, \quad \kappa=\frac{\sqrt{a} t^{\alpha / 2}}{R}, \quad \eta_{i}=R \xi_{i}
$$

we get

$$
\begin{aligned}
\bar{T} & =1-2 \sum_{i=1}^{\infty} E_{\alpha}\left(-\kappa^{2} \eta_{i}^{2}\right) \frac{J_{0}\left(\rho \eta_{i}\right)}{\eta_{i} J_{1}\left(\eta_{i}\right)} \\
\bar{\sigma}_{r r} & =-2 \sum_{i=1}^{\infty} E_{\alpha}\left(-\kappa^{2} \eta_{i}^{2}\right)\left[\frac{1}{\eta_{i}^{2}}-\frac{J_{1}\left(\rho \eta_{i}\right)}{\rho \eta_{i}^{2} J_{1}\left(\eta_{i}\right)}\right], \\
\bar{\sigma}_{\theta \theta} & =-2 \sum_{i=1}^{\infty} E_{\alpha}\left(-\kappa^{2} \eta_{i}^{2}\right)\left[\frac{1}{\eta_{i}^{2}}+\frac{J_{1}\left(\rho \eta_{i}\right)-\rho \eta_{i} J_{0}\left(\rho \eta_{i}\right)}{\rho \eta_{i}^{2} J_{1}\left(\eta_{i}\right)}\right], \\
\bar{\sigma}_{z z} & =-2 \sum_{i=1}^{\infty} E_{\alpha}\left(-\kappa^{2} \eta_{i}^{2}\right)\left[\frac{2}{\eta_{i}^{2}}-\frac{J_{0}\left(\rho \eta_{i}\right)}{\eta_{i} J_{1}\left(\eta_{i}\right)}\right] .
\end{aligned}
$$

The well-known solution of the classical thermoelasticty problem [37] is obtained when $E_{1}\left(-\kappa^{2} \eta_{i}^{2}\right)=$ $\exp \left(-\kappa^{2} \eta_{i}^{2}\right)$. The limiting case $\alpha \rightarrow 0$ with $E_{0}\left(-\kappa^{2} \eta_{i}^{2}\right)=\frac{1}{1+\kappa^{2} \eta_{i}^{2}}$ corresponds to the Helmholtz equation and to the so called "localized thermoelasticity", while the limiting case $\alpha \rightarrow 2$ with $E_{2}\left(-\kappa^{2} \eta_{i}^{2}\right)=\cos \left(\kappa \eta_{i}\right)$ corresponds to the wave equation and to the thermoelasticity without energy dissipation proposed by Green and Naghdi [40].

The results of numerical calculations are presented in Figs. 1, 2, 3, 4, 5, 6, 7, 8, 9 for typical values of the parameter $\kappa$. Figures 1, 2, 3 describe the situation typical for $0<\kappa<1$, Figs. 4, 5, 6 for $\kappa=1$, and Figs. 7, 8,9 for $1<\kappa<2$.

\section{Solution to the first Neumann problem}

In this section, we consider the heat conduction Eq. (15) under zero initial conditions (16) and (17) and the prescribed normal derivative of temperature at the boundary:

$$
r=R: \quad \frac{\partial T}{\partial r}=q(t) .
$$

Applying the Laplace transform with respect to time $t$ and the finite Hankel transform with respect to the polar coordinate $r$ (A.9) correspoding to the given boundary value of the normal derivative of temperature, we obtain

$$
T^{* *}\left(\xi_{i}, s\right)=a R J_{0}\left(R \xi_{i}\right) \frac{1}{s^{\alpha}+a \xi_{i}^{2}} q^{*}(s) .
$$

Inversion of the integral transforms leads to the following result

$$
T(r, t)=\frac{2 a}{R \Gamma(\alpha)} \int_{0}^{t}(t-\tau)^{\alpha-1} q(\tau) \mathrm{d} \tau+\frac{2 a}{R} \sum_{i=1}^{\infty} C_{\alpha, \alpha}\left(\xi_{i}, t\right) \frac{J_{0}\left(r \xi_{i}\right)}{J_{0}\left(R \xi_{i}\right)}
$$




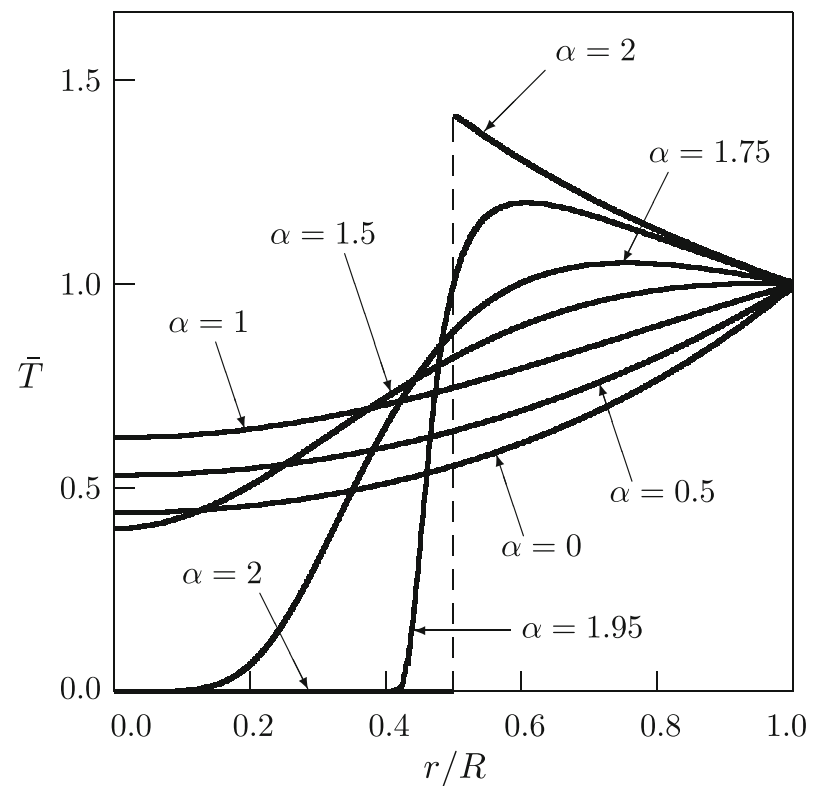

Fig. 1 Dependence of temperature on distance (the constant temperature at the boundary; $\kappa=0.5$ )

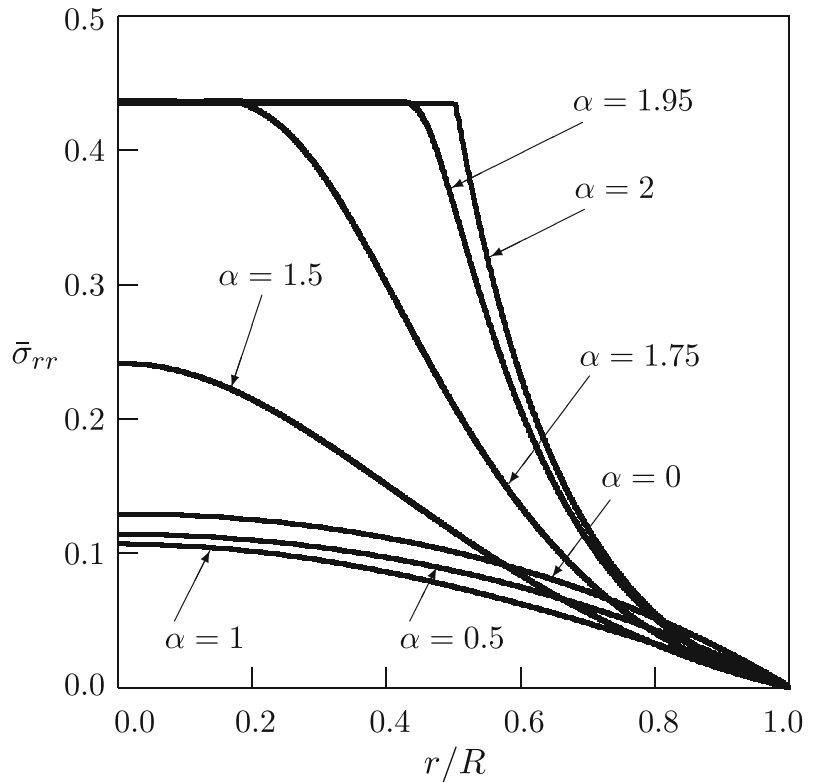

Fig. 2 Dependence of stress $\sigma_{r r}$ on distance (the constant temperature at the boundary; $\kappa=0.5$ )

$$
\begin{aligned}
& \sigma_{r r}(r, t)=-\frac{4 \mu m a}{r R} \sum_{i=1}^{\infty} C_{\alpha, \alpha}\left(\xi_{i}, t\right) \frac{J_{1}\left(r \xi_{i}\right)}{\xi_{i} J_{0}\left(R \xi_{i}\right)}, \\
& \sigma_{\theta \theta}(r, t)=\frac{4 \mu m a}{r R} \sum_{i=1}^{\infty} C_{\alpha, \alpha}\left(\xi_{i}, t\right) \frac{J_{1}\left(r \xi_{i}\right)-r \xi_{i} J_{0}\left(r \xi_{i}\right)}{\xi_{i} J_{0}\left(R \xi_{i}\right)}, \\
& \sigma_{z z}(r, t)=-\frac{4 \mu m a}{R} \sum_{i=1}^{\infty} C_{\alpha, \alpha}\left(\xi_{i}, t\right) \frac{J_{0}\left(r \xi_{i}\right)}{J_{0}\left(R \xi_{i}\right)},
\end{aligned}
$$




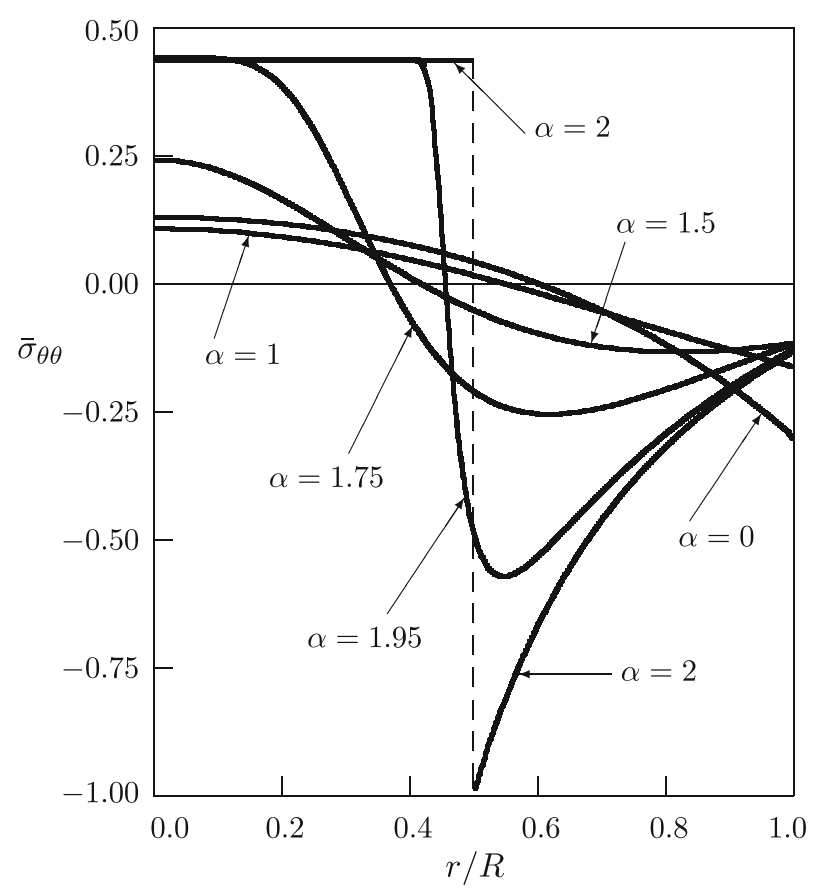

Fig. 3 Dependence of stress $\sigma_{\theta \theta}$ on distance (the constant temperature at the boundary; $\kappa=0.5$ )

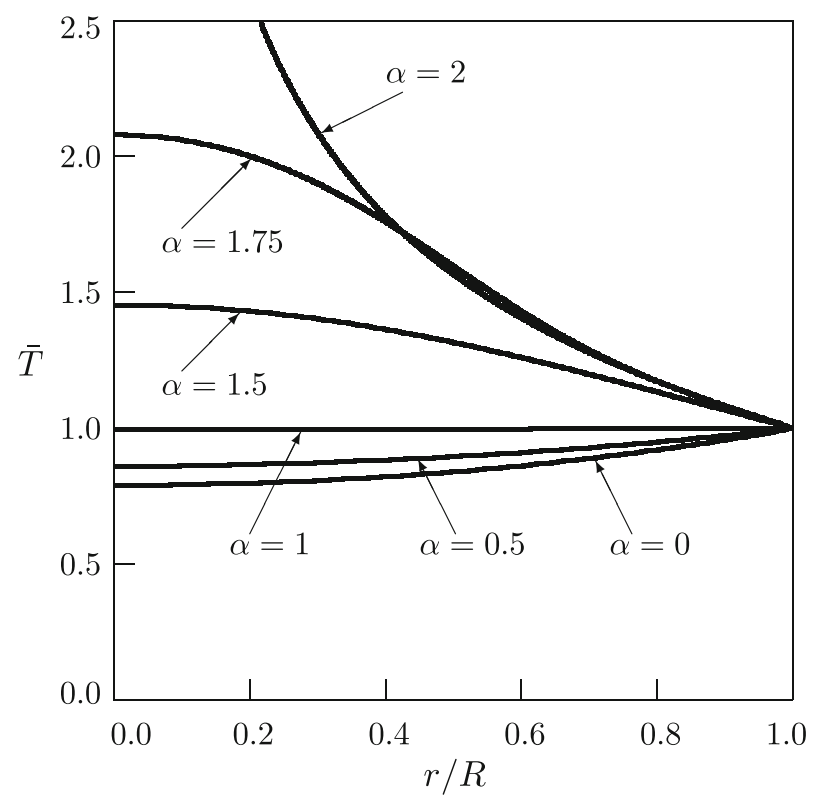

Fig. 4 Dependence of temperature on distance (the constant temperature at the boundary; $\kappa=1$ )

where

$$
C_{\alpha, \alpha}\left(\xi_{i}, t\right)=\int_{0}^{t} \tau^{\alpha-1} E_{\alpha, \alpha}\left(-a \xi_{i}^{2} \tau^{\alpha}\right) q(t-\tau) \mathrm{d} \tau .
$$

Now we consider the particular case of the obtained solution corresponding to the constant normal derivative of temperature at the boundary:

$$
r=R: \quad \frac{\partial T}{\partial r}=q_{0}=\text { const. }
$$




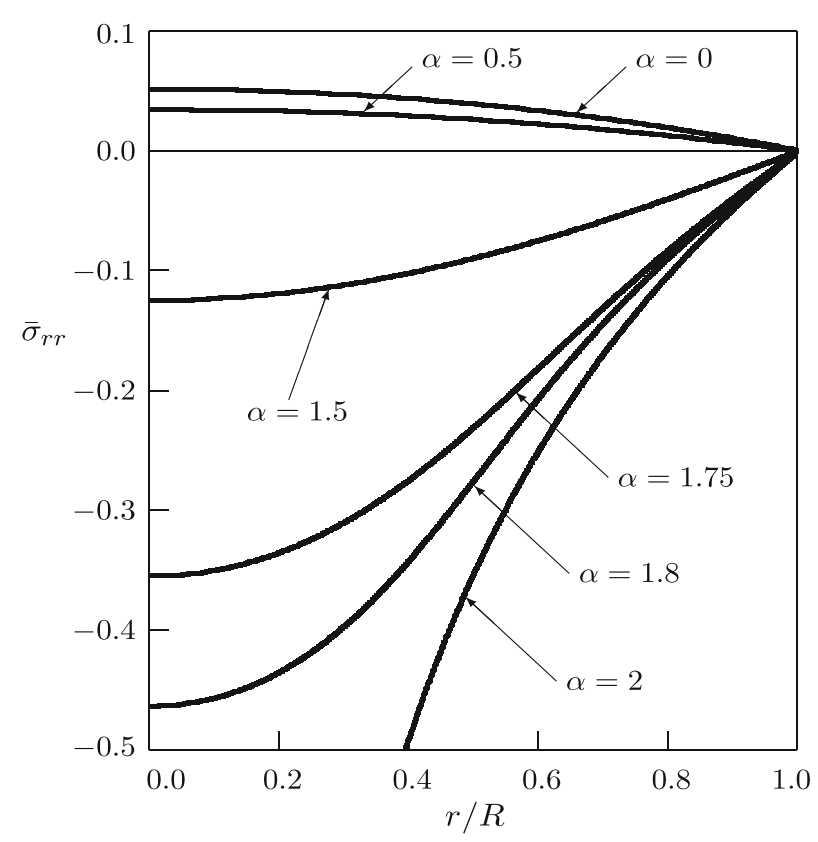

Fig. 5 Dependence of stress $\sigma_{r r}$ on distance (the constant temperature at the boundary; $\kappa=1$ )

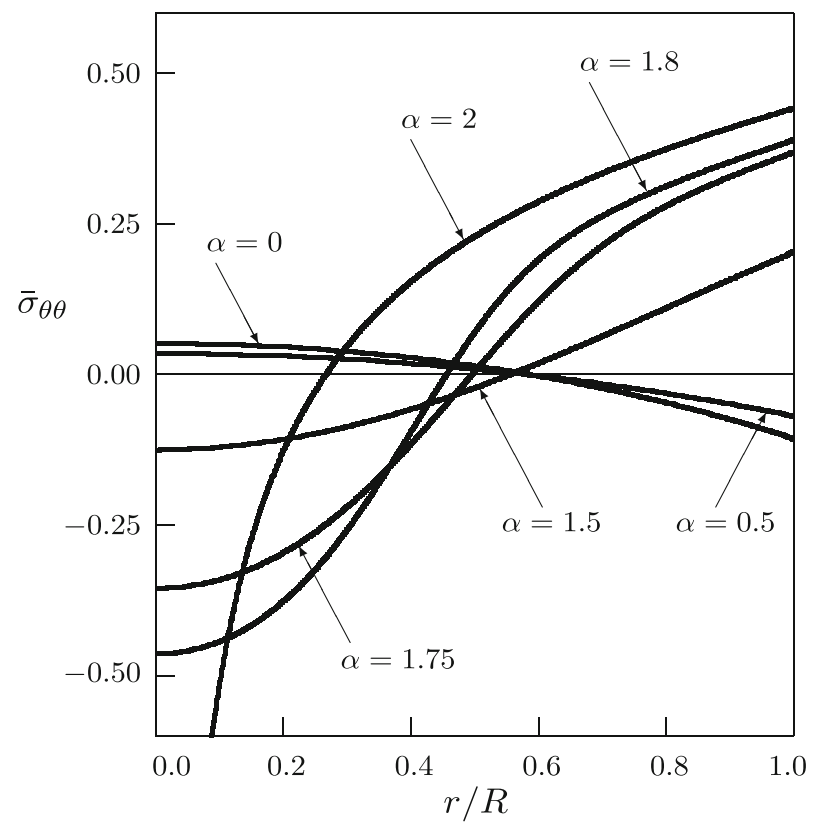

Fig. 6 Dependence of stress $\sigma_{\theta \theta}$ on distance (the constant temperature at the boundary; $\kappa=1$ )

Then

$$
T^{* *}\left(\xi_{i}, s\right)=a R q_{0} J_{0}\left(R \xi_{i}\right) \frac{1}{s\left(s^{\alpha}+a \xi_{i}^{2}\right)}
$$

and

$$
T(r, t)=\frac{2 q_{0} a t^{\alpha}}{R \Gamma(1+\alpha)}+\frac{q_{0}}{R}\left[\frac{r^{2}}{2}-\frac{R^{2}}{4}-2 \sum_{i=1}^{\infty} E_{\alpha}\left(-a \xi_{i}^{2} t^{\alpha}\right) \frac{J_{0}\left(r \xi_{i}\right)}{\xi_{i}^{2} J_{0}\left(R \xi_{i}\right)}\right]
$$




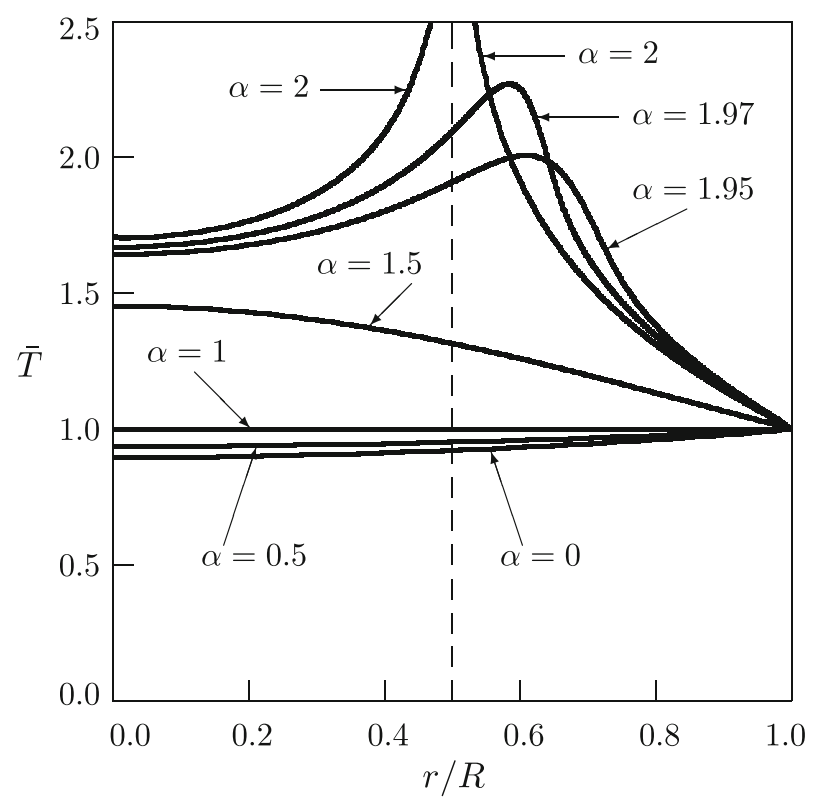

Fig. 7 Dependence of temperature on distance (the constant temperature at the boundary; $\kappa=1.5$ )

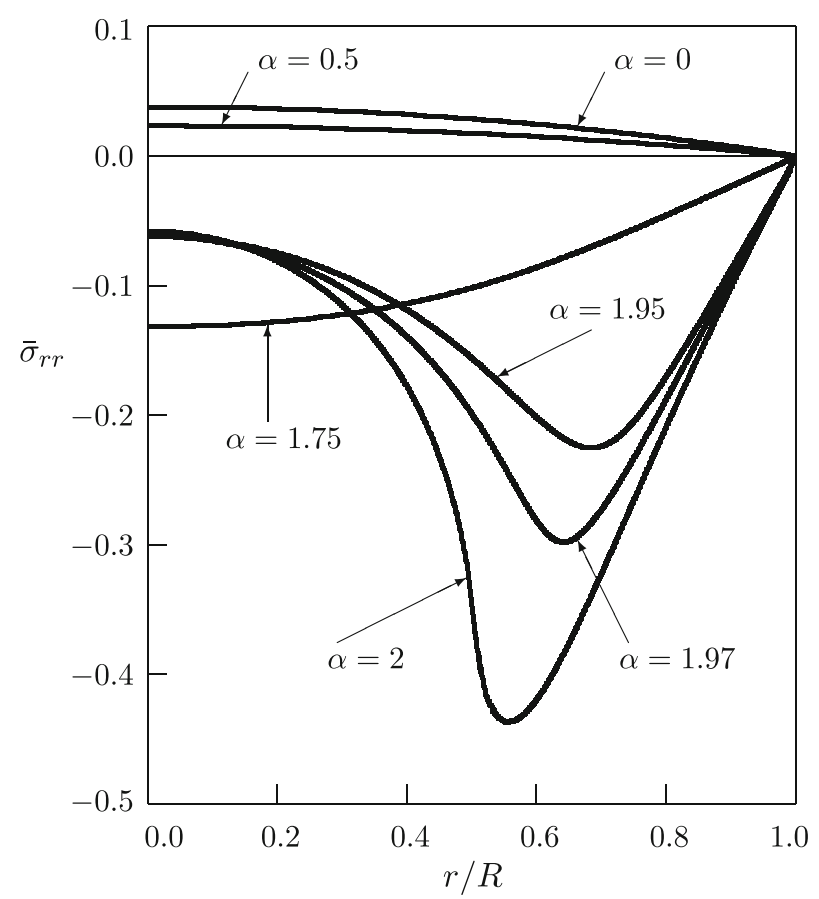

Fig. 8 Dependence of stress $\sigma_{r r}$ on distance (the constant temperature at the boundary; $\kappa=1.5$ )

For classical heat conduction equation, when $E_{1}(-x)=\mathrm{e}^{-x}$, the solution (47) coincides with the wellknown result (see e.g. Carslaw and Jaeger [41])

$$
T(r, t)=\frac{2 q_{0} a t}{R}+\frac{q_{0}}{R}\left[\frac{r^{2}}{2}-\frac{R^{2}}{4}-2 \sum_{i=1}^{\infty} \mathrm{e}^{-a \xi_{i}^{2} t} \frac{J_{0}\left(r \xi_{i}\right)}{\xi_{i}^{2} J_{0}\left(R \xi_{i}\right)}\right] .
$$

Introducing non-dimensional quantities ( $\rho, \kappa$ and $\eta_{i}$ are the same as in (33))

$$
\bar{T}=\frac{1}{R q_{0}} T, \quad \bar{\sigma}_{i j}=\frac{1}{2 \mu m R q_{0}} \sigma_{i j},
$$




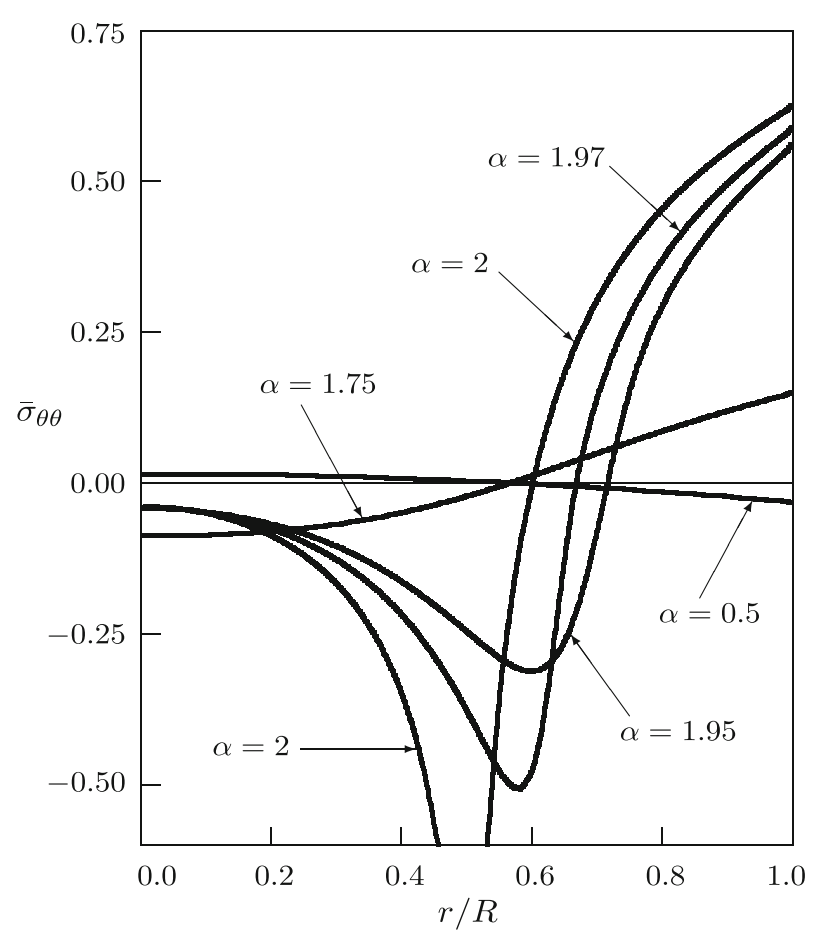

Fig. 9 Dependence of stress $\sigma_{\theta \theta}$ on distance (the constant temperature at the boundary; $\kappa=1.5$ )

we have

$$
\begin{aligned}
\bar{T} & =\frac{2 \kappa^{2}}{\Gamma(1+\alpha)}+\frac{\rho^{2}}{2}-\frac{1}{4}-2 \sum_{i=1}^{\infty} E_{\alpha}\left(-\kappa^{2} \eta_{i}^{2}\right) \frac{J_{0}\left(\rho \eta_{i}\right)}{\eta_{i}^{2} J_{0}\left(\eta_{i}\right)} \\
\bar{\sigma}_{r r} & =\frac{1}{8}\left(1-\rho^{2}\right)+2 \sum_{i=1}^{\infty} E_{\alpha}\left(-\kappa^{2} \eta_{i}^{2}\right) \frac{J_{1}\left(\rho \eta_{i}\right)}{\rho \eta_{i}^{3} J_{0}\left(\eta_{i}\right)} \\
\bar{\sigma}_{\theta \theta} & =\frac{1}{8}\left(1-3 \rho^{2}\right)-2 \sum_{i=1}^{\infty} E_{\alpha}\left(-\kappa^{2} \eta_{i}^{2}\right) \frac{J_{1}\left(\rho \eta_{i}\right)-\rho \eta_{i} J_{0}\left(\rho \eta_{i}\right)}{\rho \eta_{i}^{3} J_{0}\left(\eta_{i}\right)} \\
\bar{\sigma}_{z z} & =\frac{1}{4}-\frac{\rho^{2}}{2}+2 \sum_{i=1}^{\infty} E_{\alpha}\left(-\kappa^{2} \eta_{i}^{2}\right) \frac{J_{0}\left(\rho \eta_{i}\right)}{\eta_{i}^{2} J_{0}\left(\eta_{i}\right)}
\end{aligned}
$$

The results of numerical calculations are presented in Figs. 10, 11, 12.

\section{Solution to the second Neumann problem}

Let us investigate the heat conduction Eq. (15) under zero initial conditions (16) and (17) and the prescribed heat flux at the boundary:

$$
\begin{aligned}
& r=R: \quad D_{R L}^{1-\alpha} \frac{\partial T}{\partial r}=q(t), \quad 0<\alpha \leq 1, \\
& r=R: \quad I^{\alpha-1} \frac{\partial T}{\partial r}=q(t), \quad 1<\alpha \leq 2 .
\end{aligned}
$$

The integral transform technique results in

$$
T(r, t)=\frac{2 a}{R} \int_{0}^{t} q(\tau) \mathrm{d} \tau+\frac{2 a}{R} \sum_{i=1}^{\infty} C_{\alpha}\left(\xi_{i}, t\right) \frac{J_{0}\left(r \xi_{i}\right)}{J_{0}\left(R \xi_{i}\right)},
$$




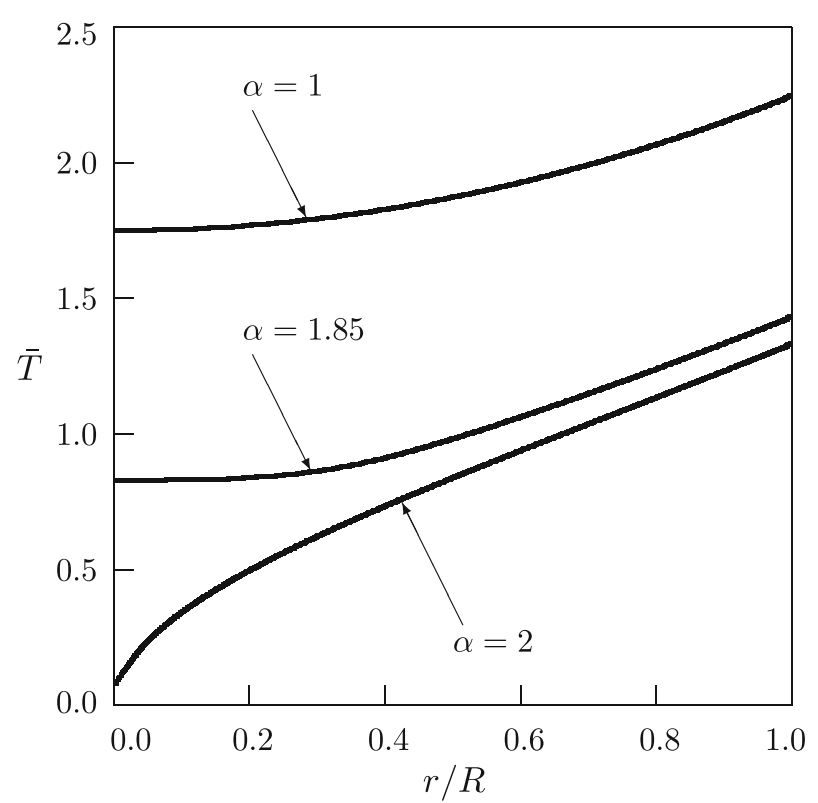

Fig. 10 Dependence of temperature on distance (the constant normal derivative of temperature at the boundary; $\kappa=1$ )

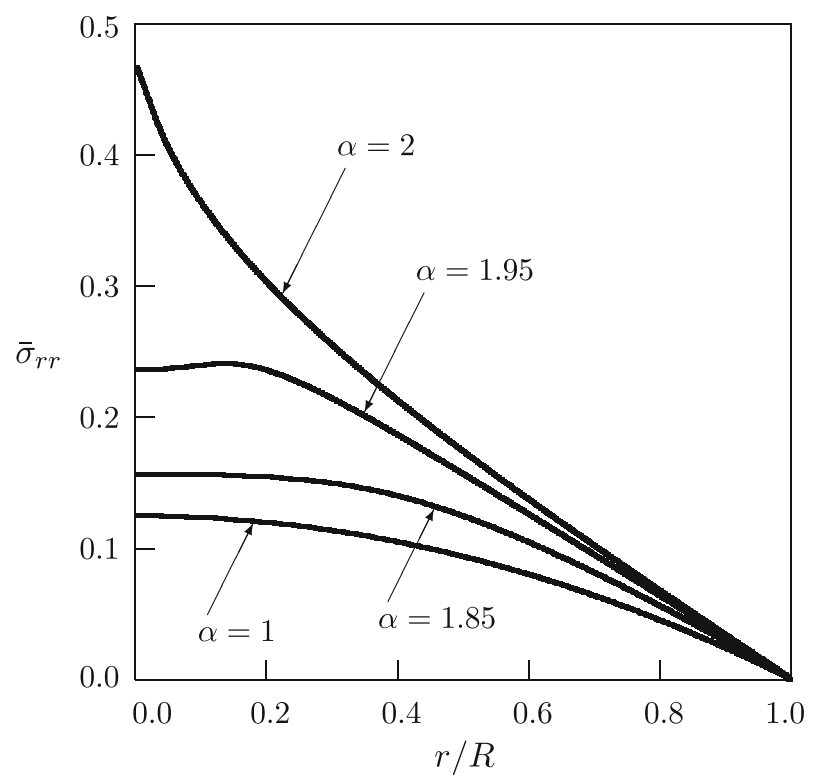

Fig. 11 Dependence of stress $\sigma_{r r}$ on distance (the constant normal derivative of temperature at the boundary; $\kappa=1$ )

$$
\begin{aligned}
& \sigma_{r r}(r, t)=-\frac{4 \mu m a}{r R} \sum_{i=1}^{\infty} C_{\alpha}\left(\xi_{i}, t\right) \frac{J_{1}\left(r \xi_{i}\right)}{\xi_{i} J_{0}\left(R \xi_{i}\right)} \\
& \sigma_{\theta \theta}(r, t)=\frac{4 \mu m a}{r R} \sum_{i=1}^{\infty} C_{\alpha}\left(\xi_{i}, t\right) \frac{J_{1}\left(r \xi_{i}\right)-r \xi_{i} J_{0}\left(r \xi_{i}\right)}{\xi_{i} J_{0}\left(R \xi_{i}\right)} \\
& \sigma_{z z}(r, t)=-\frac{4 \mu m a}{R} \sum_{i=1}^{\infty} C_{\alpha}\left(\xi_{i}, t\right) \frac{J_{0}\left(r \xi_{i}\right)}{J_{0}\left(R \xi_{i}\right)}
\end{aligned}
$$




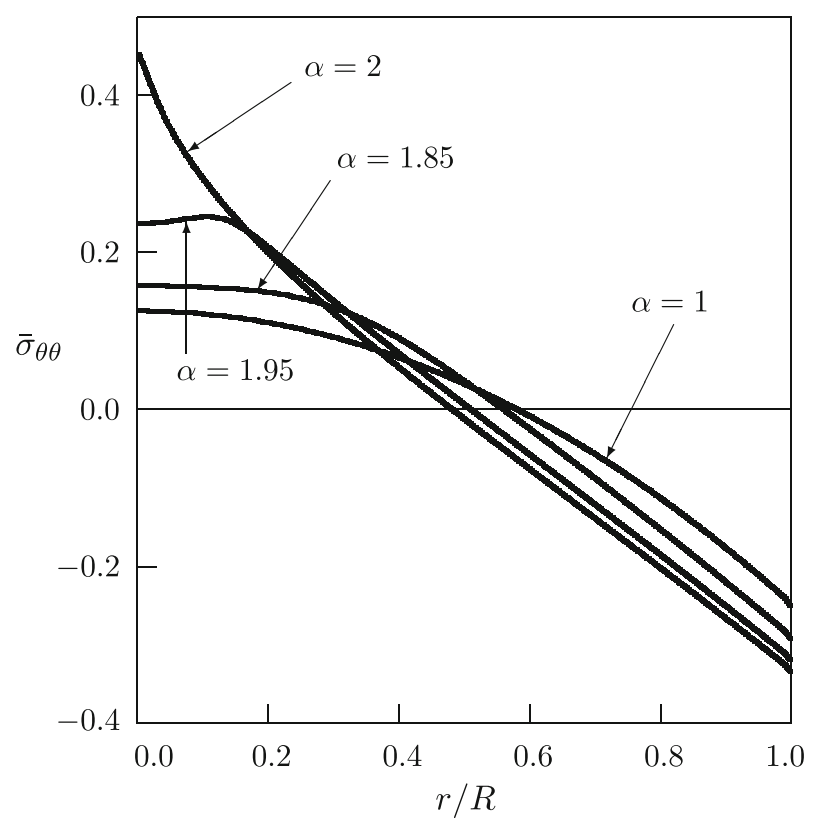

Fig. 12 Dependence of stress $\sigma_{\theta \theta}$ on distance (the constant normal derivative of temperature at the boundary; $\kappa=1$ )

where

$$
C_{\alpha}\left(\xi_{i}, t\right)=\int_{0}^{t} E_{\alpha}\left(-a \xi_{i}^{2} \tau^{\alpha}\right) q(t-\tau) \mathrm{d} \tau
$$

In the particular case corresponding to the constant heat flux at the boundary:

$$
\begin{aligned}
& r=R: \quad D_{R L}^{1-\alpha} \frac{\partial T}{\partial r}=q_{0}=\text { const }, \quad 0<\alpha \leq 1, \\
& r=R: \quad I^{\alpha-1} \frac{\partial T}{\partial r}=q_{0}=\text { const }, \quad 1<\alpha \leq 2,
\end{aligned}
$$

we get

$$
T^{* *}\left(\xi_{i}, s\right)=a R q_{0} J_{0}\left(R \xi_{i}\right) \frac{s^{\alpha-2}}{s^{\alpha}+a \xi_{i}^{2}}
$$

and

$$
T(r, t)=\frac{2 q_{0} a t}{R}+\frac{2 q_{0} a t}{R} \sum_{i=1}^{\infty} E_{\alpha, 2}\left(-a \xi_{i}^{2} t^{\alpha}\right) \frac{J_{0}\left(r \xi_{i}\right)}{J_{0}\left(R \xi_{i}\right)}
$$

For classical heat conduction equation, when $E_{1,2}(-x)=\left(1-\mathrm{e}^{-x}\right) / x$, the solution (62) with taking into account (A.15) coincides with the solution (48).

Introducing non-dimensional quantities

$$
\bar{T}=\frac{t^{\alpha-1}}{R q_{0}} T, \quad \bar{\sigma}_{i j}=\frac{t^{\alpha-1}}{2 \mu m R q_{0}} \sigma_{i j}
$$


we obtain

$$
\begin{aligned}
\bar{T} & =2 \kappa^{2}+2 \kappa^{2} \sum_{i=1}^{\infty} E_{\alpha, 2}\left(-\kappa^{2} \eta_{i}^{2}\right) \frac{J_{0}\left(\rho \eta_{i}\right)}{J_{0}\left(\eta_{i}\right)} \\
\bar{\sigma}_{r r} & =-2 \kappa^{2} \sum_{i=1}^{\infty} E_{\alpha, 2}\left(-\kappa^{2} \eta_{i}^{2}\right) \frac{J_{1}\left(\rho \eta_{i}\right)}{\rho \eta_{i} J_{0}\left(\eta_{i}\right)} \\
\bar{\sigma}_{\theta \theta} & =2 \kappa^{2} \sum_{i=1}^{\infty} E_{\alpha, 2}\left(-\kappa^{2} \eta_{i}^{2}\right) \frac{J_{1}\left(\rho \eta_{i}\right)-\rho \eta_{i} J_{0}\left(\rho \eta_{i}\right)}{\rho \eta_{i} J_{0}\left(\eta_{i}\right)} \\
\bar{\sigma}_{z z} & =-2 \kappa^{2} \sum_{i=1}^{\infty} E_{\alpha, 2}\left(-\kappa^{2} \eta_{i}^{2}\right) \frac{J_{0}\left(\rho \eta_{i}\right)}{J_{0}\left(\eta_{i}\right)}
\end{aligned}
$$

The coefficient $\kappa^{2}$ appears in Eqs. (64)-(67), as we have introduced such a non-dimensional temperature which for classical heat conduction equation coincides with that of Carslaw and Jaeger [41]. The results of numerical calculations are presented in Figs. 13, 14, 15 for $\kappa=1$.

\section{Results and discussion}

The classical Fourier law is a phenomenological law, which states the proportionality of the flux to the gradient of the transported quantity. It is met in several physical contexts with different names. It is well known that from mathematical viewpoint, the Fourier law in the theory of heat conduction and the Fick law in the theory of diffusion is indentical. The time-nonlocal dependence (1) can also be formulated in terms of the matter flux and concentration gradient. The diffusion interpretation may give a clearer insight into the physical aspects of the theory.

At the level of individual particle motions, the classical diffusion corresponds to the Brownian motion that is characterized by a mean-squared displacement increasing linearly with time. Anomalous diffusion, which is exemplified by a mean-squared displacement with the power-law time dependence and is described by diffusion equation with fractional derivative, at the level of individual particle motion has been modeled in numerous ways. For example, the continuous-time random-walk theory allows one to extend classical Brownian random walks to variable jump lengths and waiting times between successive jumps. The power-law tails

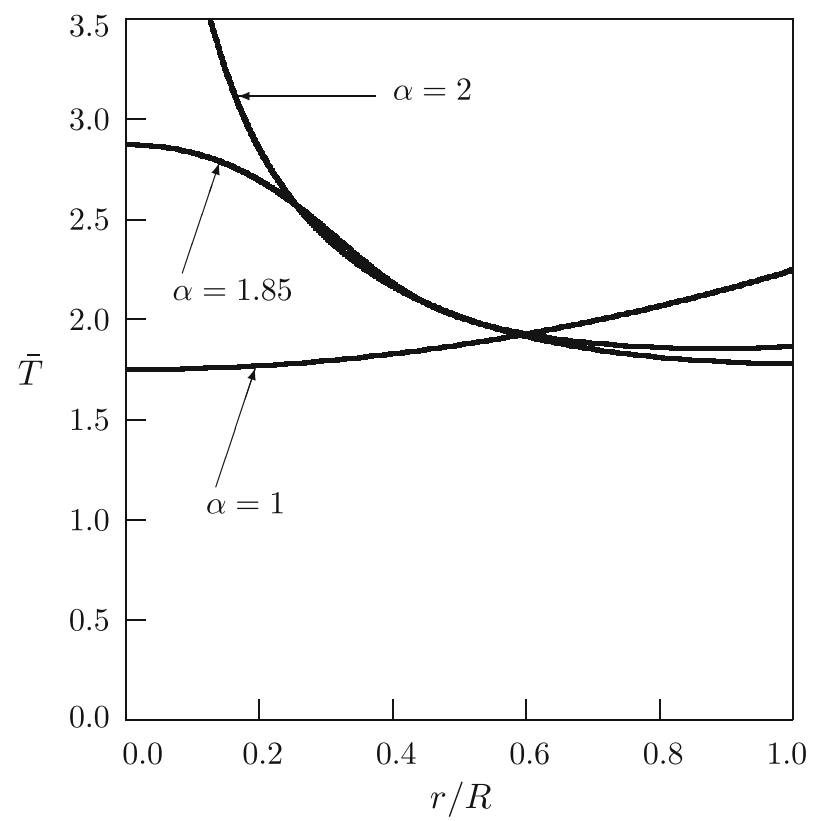

Fig. 13 Dependence of temperature on distance (the constant heat flux at the boundary; $\kappa=1$ ) 


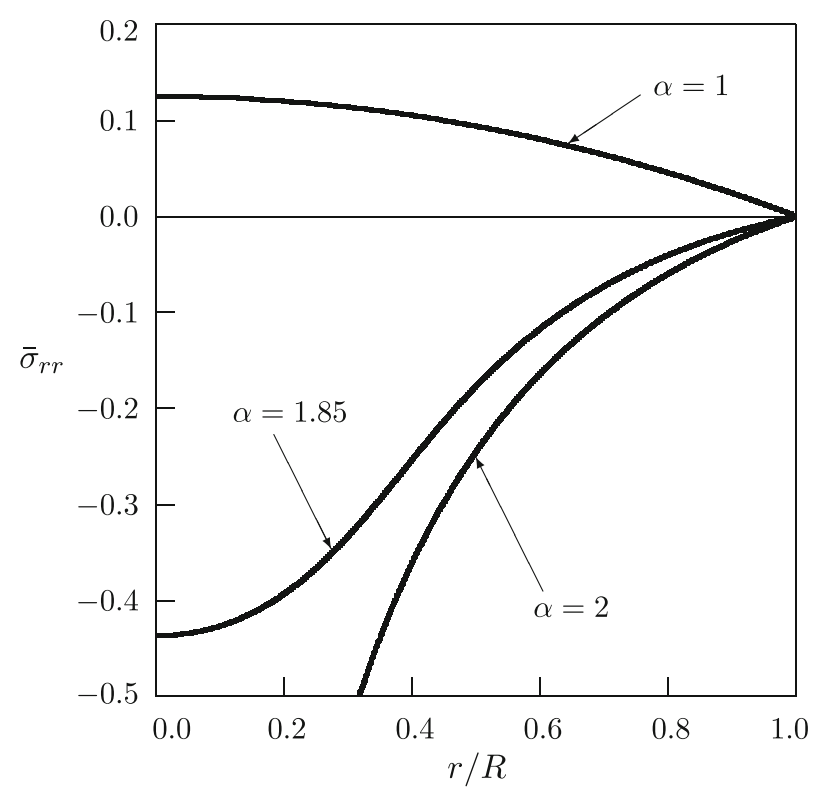

Fig. 14 Dependence of stress $\sigma_{r r}$ on distance (the constant heat flux at the boundary; $\kappa=1$ )

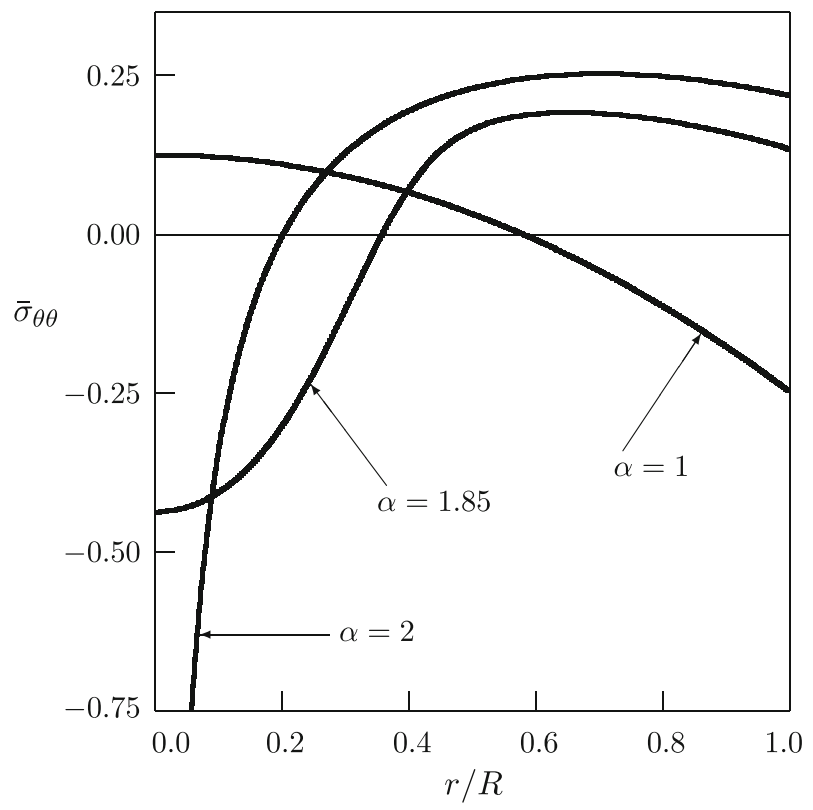

Fig. 15 Dependence of stress $\sigma_{\theta \theta}$ on distance (the constant heat flux at the boundary; $\kappa=1$ )

make it possible to have very long waiting times, and in the subdiffusion regime $(0<\alpha<1)$ particles on the average move slower than in the ordinary diffusion that corresponds to $\alpha=1$. In the superdiffusion regime $(1<\alpha<2)$, it is possible to have very long jumps, and particles on the average move faster than in the ordinary diffusion. As a rule, anomalous diffusion and anomalous heat conduction have the same origin.

In this paper, we have obtained solutions to time-fractional heat conduction equation with the Caputo timefractional derivative. These solutions reduce to the solutions of classical heat conduction equation in the limit $\alpha=1$. In the case $0<\alpha<1$, the considered equation interpolates the elliptic Helmholtz equation $(\alpha=0)$ and parabolic heat conduction equation. In the limit $\alpha \rightarrow 0$, the obtained solutions reduce to the solutions of Helmholtz equation. In the case $1<\alpha<2$, the time-fractional heat conduction equation interpolates the parabolic heat conduction equation and the hyperbolic wave equation, and the proposed theory of thermal stresses 
interpolates the classical thermoelasticity $(\alpha=1)$ and that without energy dissipation $(\alpha=2)$ introduced by Green and Naghdi [40].

The computations are carried out according to the values of parameter $\kappa$ reflecting the characteristic features of the solution for various order of the time-fractional derivative. Three distinguishing values of the parameter $\kappa$ are considered: $0<\kappa<1, \kappa=1$ and $1<\kappa<2$. For wave equation, these values correspond to three characteristic cases: the wave front does not yet arrive at the origin, the wave front arrives at the origin, and the wave front reflects from the origin. Analysis of the solution of the wave equation and the corresponding thermal stresses (in the framework of thermoelasticity without energy dissipation) shows that for $\kappa=1$, there arise singularities at the origin $\rho=0$ in temperature and all the components of the stress tensor (Figs. 4, 5, 6). For $1<\kappa<2$ such singularities appear at $\rho=\kappa-1$ (Figs. 7, 8,9) except the component $\sigma_{r r}$ (Fig. 8) that remains continuous. With $\alpha$ approaching 2 , the fractional thermoelasticity solutions exhibit propagating humps, underlining the proximity to the standard wave equation in contrast to the shape of curves describibg the heat subdiffusion regime $0<\alpha<1$.

For reasons of space, in calculations concerning the Neumann problem, we have restricted ourselves to the characteristic case $\kappa=1$ (see Figs. 10, 11, 12, 13, 14, 15). In these figures, we have not displayed curves for $0<\alpha<1$, as the solutions for such values of $\alpha$ are very close to those for $\alpha=1$. In the case of constant normal derivative, the temperature at the origin is less than temperature at a surface of a cylinder for all values of $\alpha$, but in the case of constant heat flux, the temperature at the origin for $1<\alpha \leq 2$ is larger than that at a surface. In the case of ballistic heat conduction $(\alpha=2)$, the temperature as well as the stress components have singularity at the origin. In the case of the first Neumann problem, the stress component $\sigma_{r r}$ is positive for all $0<\alpha \leq 2$, but in the case of the second Neumann problem, the component $\sigma_{r r}$ is positive for $0<\alpha<1$ and becomes negative for fast heat conduction characterized by $1<\alpha \leq 2$. In the case of constant normal derivative, the behavior of the stress component $\sigma_{\theta \theta}$ is similar for all values of $0<\alpha \leq 2$ (Fig. 12). Situation changes radically in the case of constant heat flux at the bounadry: stresses $\sigma_{\theta \theta}$ in the cases $0<\alpha \leq 1$ and $1<\alpha \leq 2$ have different signs (Fig. 15).

\section{Conclusions}

We have investigated axisymmetric thermal stresses in a cylinder in a frame-work of quasi-static uncoupled theory of thermoelasticity based on fractional heat conduction equation with the Caputo time-fractional derivative of order $0<\alpha<2$. New analytical solutions have been derived for three types of boundary conditions: the given boundary value of temperature, the given boundary value of the normal derivative of temperature, and the prescribed heat flux expressed in terms of time-fractional integrals and derivatives of normal derivative of temperature. The numerical results demonstrate the significant influence of the order of time derivative on the temperature distribution as well as on the stress distribution with radial coordinate. In this paper, we have restricted ourselves to axisymmetric case. In the future work, fractional heat conduction and associated thermal stresses in a cylinder in the case of dependence of temperature on three spatial coordinates merit consideration. The first step in this direction has been made in [42].

Open Access This article is distributed under the terms of the Creative Commons Attribution Noncommercial License which permits any noncommercial use, distribution, and reproduction in any medium, provided the original author(s) and source are credited.

\section{Appendix}

Here, we recall the properties of finite Hankel transforms (see Sneddon [43]). The finite Hankel transform of order zero is defined as

$$
\mathcal{H}_{D}\{f(r)\}=f^{*}\left(\xi_{i}\right)=\int_{0}^{R} r f(r) J_{0}\left(r \xi_{i}\right) \mathrm{d} r
$$


having the inverse

$$
\mathcal{H}_{D}^{-1}\left\{f^{*}\left(\xi_{i}\right)\right\}=f(r)=\frac{2}{R^{2}} \sum_{i=1}^{\infty} f^{*}\left(\xi_{i}\right) \frac{J_{0}\left(r \xi_{i}\right)}{\left[J_{1}\left(R \xi_{i}\right)\right]^{2}}
$$

with the sum over all positive roots of transcendental equation

$$
J_{0}\left(R \xi_{i}\right)=0 .
$$

We have introduced notation with the index $D$ to emphasize that this transform is used for solving problems under the Dirichlet boundary condition with the prescribed value of a function according to the following rule

$$
\mathcal{H}_{D}\left\{\frac{\mathrm{d}^{2} f}{\mathrm{~d} r^{2}}+\frac{1}{r} \frac{\mathrm{d} f}{\mathrm{~d} r}\right\}=-\xi_{i}^{2} \mathcal{H}_{D}\{f(r)\}+R \xi_{i} J_{1}\left(R \xi_{i}\right) f(R) .
$$

Since

$$
\int_{0}^{R} r J_{0}\left(r \xi_{i}\right) \mathrm{d} r=\frac{R}{\xi_{i}} J_{1}\left(R \xi_{i}\right)
$$

and

$$
\int_{0}^{R}\left(R^{2}-r^{2}\right) r J_{0}\left(r \xi_{i}\right) \mathrm{d} r=\frac{4 R}{\xi_{i}^{3}} J_{1}\left(R \xi_{i}\right)-\frac{2 R^{2}}{\xi_{i}^{2}} J_{0}\left(R \xi_{i}\right),
$$

we have

$$
\frac{2}{R} \sum_{i=1}^{\infty} \frac{1}{\xi_{i}} \frac{J_{0}\left(r \xi_{i}\right)}{J_{1}\left(R \xi_{i}\right)}=1
$$

and

$$
\frac{2}{R} \sum_{i=1}^{\infty} \frac{1}{\xi_{i}^{3}} \frac{J_{0}\left(r \xi_{i}\right)}{J_{1}\left(R \xi_{i}\right)}=\frac{1}{4}\left(R^{2}-r^{2}\right) .
$$

Another type of the finite Hankel transform of order zero

$$
\mathcal{H}_{N}\{f(r)\}=f^{*}\left(\xi_{i}\right)=\int_{0}^{R} r f(r) J_{0}\left(r \xi_{i}\right) \mathrm{d} r
$$

with the inverse

$$
\mathcal{H}_{N}^{-1}\left\{f^{*}\left(\xi_{i}\right)\right\}=f(r)=\frac{2}{R^{2}} \sum_{i=0}^{\infty} f^{*}\left(\xi_{i}\right) \frac{J_{0}\left(r \xi_{i}\right)}{\left[J_{0}\left(R \xi_{i}\right)\right]^{2}}
$$

is obtained when $\xi_{i}$ are the non-negative roots of transcendental equation

$$
J_{1}\left(R \xi_{i}\right)=0 .
$$

The index $N$ indicates that this integral transform is used for solving problems under the Neumann boundary condition with the prescribed value of the normal derivative of a function according to the following rule

$$
\mathcal{H}_{N}\left\{\frac{\mathrm{d}^{2} f}{\mathrm{~d} r^{2}}+\frac{1}{r} \frac{\mathrm{d} f}{\mathrm{~d} r}\right\}=-\xi_{i}^{2} \mathcal{H}_{N}\{f(r)\}+R J_{0}\left(R \xi_{i}\right)\left(\frac{\mathrm{d} f}{\mathrm{~d} r}\right)_{r=R} .
$$


As

$$
\int_{0}^{R}\left(R^{2}-r^{2}\right) r \mathrm{~d} r=f^{*}(0)=\frac{1}{4} R^{4}
$$

and

$$
\int_{0}^{R}\left(R^{2}-r^{2}\right) r J_{0}\left(r \xi_{i}\right) \mathrm{d} r=-\frac{2 R^{2}}{\xi_{i}^{2}} J_{0}\left(R \xi_{i}\right), \quad i \geq 1
$$

we get

$$
2 \sum_{i=1}^{\infty} \frac{1}{\xi_{i}^{2}} \frac{J_{0}\left(r \xi_{i}\right)}{J_{0}\left(R \xi_{i}\right)}=\frac{1}{2} r^{2}-\frac{1}{4} R^{2}
$$

and

$$
2 \sum_{i=1}^{\infty} \frac{1}{\xi_{i}^{3}} \frac{J_{1}\left(r \xi_{i}\right)}{J_{0}\left(R \xi_{i}\right)}=\frac{r}{8}\left(r^{2}-R^{2}\right) .
$$

\section{References}

1. Podstrigach, Y.S., Kolyano, Y.M.: Generalized Thermomechanics. Naukova Dumka, Kiev (1976) (in Russian)

2. Chandrasekharaiah, D.S.: Thermoelasticity with second sound: a review. Appl. Mech. Rev. 39, 355-376 (1986)

3. Ignaczak, J.: Generalized thermoelasticity and its applications. In: Hetnarski, R.B. (ed.) Thermal Stresses III, pp. $279-354$. North-Holland, Amsterdam (1989)

4. Chandrasekharaiah, D.S.: Hiperbolic thermoelasticity: a review of recent literature. Appl. Mech. Rev. 51, $705-729$ (1998)

5. Tamma, K.K., Zhou, X.: Macroscale and microscale thermal transport and thermo-mechanical interactions: Some noteworthy perspectives. J. Therm. Stress. 21, 405-449 (1998)

6. Hetnarski, R.B., Ignaczak, J.: Generalized thermoelasticity. J. Therm. Stress. 22, 451-476 (1999)

7. Ignaczak, J., Ostoja-Starzewski, M.: Thermoelasticity with Finite Wave Speeds. Oxford University Press, Oxford (2010)

8. Sherief, H.H., Anwar, M.N.: A problem in generalized thermoelasticity for an infinitely long annular cylinder composed of two different materials. Acta Mech. 80, 137-149 (1989)

9. Ezzat, M.: Fundamental solution in thermoelasticity with two relaxation times for cylindrical regions. Int. J. Eng. Sci. 33, 2011-2020 (1995)

10. Mukhopadhyay, S., Mukherjee, R.N.: Thermoelastic interaction in a transversally isotropic cylinder subjected to ramp type increase in boundary temperature and load. Indian J. Pure Appl. Math. 33, 635-646 (2002)

11. Sherief, H.H., Elmisiery, A.E.M., Elhagary, M.A.: Generalized thermoelastic problem for an infinitely long hollow cylinder for short times. J. Therm. Stress. 27, 885-902 (2004)

12. He, T., Tian, X., Shen, Y.: A generalized electromagneto-thermoelastic problem for an infinitely long solid cylinder. Eur. J. Mech. A/Solids 24, 349-359 (2005)

13. El-Bary, A.A.: An infinite thermoelastic long annular cylinder with variable thermal conductivity. J. Appl. Sci. Res. 2, $341-345$ (2006)

14. Aouadi, M.: A generalized thermoelastic diffusion problem for an infinitely long solid cylinder. Int. J. Math. Math. Sci. Article ID 25976, 1-15 (2006)

15. Bagri, A., Eslami, M.R.: A unified generalized thermoelasticity formulation; application to thick functionally graded cylinders. J. Therm. Stress. 30, 911-930 (2007)

16. Bagri, A., Eslami, M.R.: A unified generalized thermoelasticity; solution for cylinders and spheres. Int. J. Mech. Sci. 49, 1325-1335 (2007)

17. Kar, A., Kanoria, M.: Thermoelastic interaction with energy dissipation in a transversely isotropic thin circular disc. Eur. J. Mech. A/Solids 26, 969-981 (2007)

18. Youssef, H.M., Abbas, I.A.: Thermal shock problem of generalized thermoelasticity for an infinitely long annular cylinder with variable thermal conductivity. Comput. Methods Sci. Technol. 13, 95-100 (2007)

19. Mukhopadhyay, S., Kumar, R.: Solution of a problem of generalized thermoelasticity of an annular cylinder with variable material properties by finite difference method. Comput. Methods Sci. Technol. 15, 169-176 (2009)

20. Povstenko, Y.Z.: Fractional heat conduction equation and associated thermal stress. J. Therm. Stress. 28, 83-102 (2005)

21. Povstenko, Y.Z.: Fractional heat conduction equation and associated thermal stresses in an infinite solid with spherical cavity. Q. J. Mech. Appl. Math. 61, 523-547 (2008)

22. Povstenko, Y.: Thermoelasticity which uses fractional heat conduction equation. J. Math. Sci. 162, 296-305 (2009)

23. Povstenko, Y.Z.: Theory of thermoelasticity based on the space-time-fractional heat conduction equation. Phys. Scr. T 136, 014017 (6 pp) (2009) 
24. Povstenko, Y.Z.: Two-dimensional axisymmetric stresses exerted by instantaneous pulses and sources of diffusion in an infinite space in a case of time-fractional diffusion equation. Int. J. Solids Struct. 44, 2324-2348 (2007)

25. Povstenko, Y.Z.: Fractional radial heat conduction in an infinite medium with a cylindrical cavity and associated thermal stress. Mech. Res. Commun. 37, 436-440 (2010)

26. Povstenko, Y.Z.: Fractional radial diffusion in a cylinder. J. Mol. Liq. 137, 46-50 (2008)

27. Narahari Achar, B.N., Hanneken, J.W.: Fractional radial diffusion in a cylinder. J. Mol. Liq. 114, 147-151 (2004)

28. Özdemir, N., Karadeniz, D.: Fractional diffusion-wave problem in cylindrical coordinates. Phys. Lett. A 372, 5968-5972 (2008)

29. Özdemir, N., Karadeniz, D., Iskender, B.B.: Fractional optimal control problem of a distributed system in cylindrical coordinates. Phys. Lett. A 373, 221-226 (2009)

30. Özdemir, N., Agrawal, O.P., Karadeniz, D., Iskender, B.B.: Fractional optimal control problem of an axis-symmetric diffusion-wave propagation. Phys. Scr. T 136, 014024 (5 pp) (2009)

31. Lenzi, E.K., da Silva, L.R., Silva, A.T., Evangelista, L.R., Lenzi, M.K.: Some results for a fractional diffusion equation with radial symmetry in a confined region. Phys A 388, 806-810 (2009)

32. Qi, H., Liu, J.: Time-fractional radial diffusion in hollow geometries. Meccanica 45, 577-583 (2010)

33. Gorenflo, R., Mainardi, F. : Fractional calculus: integral and differential equations of fractional order. In: Carpinteri, A., Mainardi, F. (eds.) Fractals and Fractional Calculus in Continuum Mechanics, pp. 223-276. Springer, New York (1997)

34. Kilbas, A., Srivastava, H.M., Trujillo, J.J.: Theory and Applications of Fractional Differential Equations. Elsevier, Amsterdam (2006)

35. Nowacki, W.: Thermoelasticity. PWN—Polish Scientific Publishers, Warszawa (1986)

36. Parkus, H.: Instationäre Wärmespannungen. Springer, Wien (1959)

37. Noda, N., Hetnarski, R., Tanigawa, Y.: Thermal Stresses, 2nd edn. Taylor \& Francis, New York (2003)

38. Podlubny, I.: Fractional Differential Equations. Academic Press, San Diego (1999)

39. Erdélyi, A., Magnus, W., Oberhettinger, F., Tricomi, F.: Higher Transcendental Functions, vol. 3. McGraw-Hill, New York (1955)

40. Green, A.E., Naghdi, P.M.: Thermoelasticity without energy dissipation. J. Elast. 31, 189-208 (1993)

41. Carslaw, H.S., Jaeger, J.C.: Conduction of Heat in Solids, 2nd edn. Oxford University Press, Oxford (1959)

42. Povstenko, Y.Z.: Non-axisymmetric solutions to time-fractional diffusion-wave equation in an infinite cylinder. Frac. Calc. Appl. Anal. 14, 418-435 (2011). doi:10.2478/s13540-011-0026-4

43. Sneddon, I.N.: The Use of Integral Transforms. McGraw-Hill, New York (1972) 